\title{
A REMARK ON IRREDUCIBLE SPACES
}

\section{J. C. SMITH}

\begin{abstract}
ABSTRACr. A topological space $X$ is called irreducible if every open cover of $X$ has an open refinement which covers $X$ minimally. In this paper we show that weak $\bar{\theta}$-refinable spaces are irreducible. A modification of the proof of this result then yields that $\aleph_{1}$-compact, weak $\overline{\delta \theta}$-refinable spaces are Lindelöf. It then follows that perfect, $\aleph_{1}$-compact weak $\delta \theta$-refinable spaces are irreducible. A number of known results follow as corollaries.
\end{abstract}

1. Introduction. An open cover $\mathcal{G}$ of a topological space $X$ is called minimal provided no proper subcollection of $\mathcal{G}$ covers $X$. Recently there has been renewed interest in determining which types of open covers have minimal open refinements. A topological space $X$ is called irreducible if every open cover of $X$ has a minimal open refinement.

In 1950, R. Arens and J. Dugundji [2] showed that metacompact spaces were irreducible and from this showed that a space is compact if and only if it is countably compact and metacompact. In 1965, J. Worrell and H. Wicke [12] stated without proof that $\theta$-refinable spaces are irreducible, and in 1972, U. Christian [9], [10] investigated spaces of minimal cover refinable type and provided a simple proof that subparacompact spaces are irreducible. In [6], [7] J. Boone gave a proof for the above result of Worrell and Wicke and then extended this technique using an involved argument to show that weak $\bar{\theta}$ refinable spaces, introduced by the author in [11], are irreducible.

In $\$ 2$ of this paper we investigate the conditions under which certain $F_{\sigma}$ subsets of a topological space have minimal open covers. As an application of this we provide a relatively simple constructive proof of Boone's second result.

In $\$ 3$ we give conditions which ensure that weak $\overline{\delta \theta}$-refinable and weak $\delta \theta$ refinable spaces are Lindelöf and irreducible. In particular, we show that $\boldsymbol{\aleph}_{1^{-}}$ compact, weak $\overline{\delta \theta}$-refinable spaces are Lindelöf. Various open questions are listed in $\$ 4$.

The following notation and definitions are included for the benefit of the reader.

Definition 1.1. A space $X$ is called a weak $\bar{\theta}$-refinable space provided every open cover $\mathcal{G}$ of $X$ has a refinement $\cup_{i=1}^{\infty} \mathcal{G}_{i}$ satisfying:

(i) each $\mathcal{G}_{i}=\left\{G(\alpha, i): \alpha \in A_{i}\right\}$ is an open collection of subsets of $X$;

(ii) for each $x \in X$, there exists an $n(x)$ such that $0<$ ord $\left(x, \mathcal{G}_{n(x)}\right)<\infty$;

Presented to the Society, Auguest 22, 1975; received by the editors May 10, 1975 and, in revised form, October 14, 1975.

AMS (MOS) subject classifications (1970). Primary 54D20; Secondary 54A25.

Key words and phrases. Metacompact, $\theta$-refinable, weak $\bar{\theta}$-refinable, $\delta \theta$-refinable, weak $\overline{\delta \theta}$ refinable, $\aleph_{1}$-compact, compact, countably compact, Lindelöf, minimal cover, irreducible, maximal distinguished set, perfect.

- American Mathematical Society 1976 
(iii) $\left\{G_{i}^{*}=\cup_{\alpha \in A_{i}} G(\alpha, i)\right\}_{i=1}^{\infty}$ is point finite.

An open cover $\cup_{i=1}^{\infty} \mathcal{G}_{i}$ satisfying (i)-(iii) above is called a weak $\bar{\theta}$-cover.

H. R. Bennett and D. J. Lutzer introduced the following [4].

DEFINITION 1.2. A space $X$ is called weak $\theta$-refinable if every open cover $\mathcal{G}$ of $X$ has a refinement $\cup_{i=1}^{\infty} \mathcal{G}_{i}$ satisfying properties (i) and (ii) of Definition 1.1 above. Such covers are called weak $\theta$-covers.

It is known that $\theta$-refinable $\Rightarrow$ weak $\bar{\theta}$-refinable $\Rightarrow$ weak $\theta$-refinable [11] and that neither implication is reversible.

Throughout this paper we will use the notation that if $\mathcal{G}_{i}=\left\{G_{\alpha}: \alpha \in A_{i}\right\}$ is a collection of sets, then $G_{i}^{*}=\cup_{\alpha \in A_{i}} G_{\alpha}$ and $\mathcal{G}^{*}=\left\{G_{i}^{*}\right\}_{i=1}^{\infty}$.

All spaces are assumed to be $T_{1}$.

The following, Theorem 1.1 of [7], is referred to in several proofs and hence is included for the benefit of the reader.

THEOREM 1.3. A space $X$ is irreducible if and only if for each open cover $\left\{V_{\alpha}: \alpha \in A\right\}$ of $X$ there exists a discrete collection of nonempty closed sets $\left\{T_{\beta}: \beta \in B\right\}$ with $B \subseteq A$ such that (i) $T_{\beta} \subseteq V_{\beta}$ for each $\beta \in B$ and (ii) $\left\{V_{\beta}: \beta \in B\right\}$ covers $X$.

COROLlaRY 1.4. If $\mathfrak{V}=\left\{V_{\alpha}: \alpha \in A\right\}$ is a minimal cover of $X$, then there exists a discrete collection $\mathscr{D}=\left\{D_{\alpha}: \alpha \in A\right\}$ of nonempty closed subsets of $X$ such that

(i) $D_{\alpha} \subseteq V_{\alpha}$ for each $\alpha \in A$ and

(ii) $D_{\alpha} \cap V_{\beta}=\varnothing$ for $\alpha \neq \beta$.

2. The irreducibility of weak $\bar{\theta}$-refinable spaces. In [6] J. Boone showed that every $\boldsymbol{\theta}$-refinable space was irreducible and later in [7] showed that weak $\overline{\boldsymbol{\theta}}$ refinable spaces also had this property.

By first observing several properties of minimal covers we present an alternate proof of Boone's result. The technique used in this development will later be modified to obtain analogous results.

THEOREM 2.1. Let $F=\cup_{i=1}^{\infty} F_{i}$ be an $F_{\sigma}$-subset of a space $X$ and let $\mathfrak{V}=\cup_{i=1}^{\infty} \mathcal{V}_{i}$ be a sequence of collections of open subsets of $X$ such that each $\mathfrak{V}_{i}$ is a minimal cover of $H_{i}=F_{i}-\cup_{j<i} V_{j}^{*}$ where $V_{j}^{*}=\cup\left\{V: V \in \mathfrak{V}_{j}\right\}$.

Then $\mathcal{T}$ has an open (in $X)$ refinement which covers $F$ minimally.

Proof. Let $\mathscr{V}_{i}=\left\{V(\alpha, i): \alpha \in A_{i}\right\}$ for each $i$. Since $\mathscr{V}_{i}$ is a minimal cover of $H_{i}$, by Corollary 1.4 there exists a discrete collection $\mathscr{D}_{i}=\left\{D(\alpha, i): \alpha \in A_{i}\right\}$ of nonempty closed subsets of $H_{i}$ such that

(i) $D(\alpha, i) \subseteq V(\alpha, i)$ for each $\alpha \in A_{i}$, and

(ii) $D(\alpha, i) \cap V(\beta, i)=\varnothing$ for $\alpha \neq \beta$.

Define for each $i, \mho_{i}=\left\{W(\alpha, i)=V(\alpha, i)-\cup_{j<i} F_{j}: \alpha \in A_{i}\right\}$ and let $=\cup_{i=1}^{\infty} \mho_{i}$.

We assert that $थ$ is the desired minimal open cover of $F$. It is easy to check that $\mathscr{W}$ is an open refinement which covers $F$. Since $D(\alpha, i) \subseteq W(\alpha, i)$ $\subseteq V(\alpha, i)$ for each $\alpha \in A_{i}, D(\alpha, i) \cap W(\beta, i)=\varnothing$ for $\beta \neq \alpha$. Also for $i$ $<j, D(\alpha, i) \subseteq H_{i} \subseteq F_{i}$ so that $D(\alpha, i) \cap W(\beta, j)=\varnothing$ for all $\beta \in A_{j}$. Likewise for $j<i, W(\beta, j) \subseteq V_{j}^{*}$ and hence $D(\alpha, i) \cap W(\beta, j)=\varnothing$ for all $\beta \in A_{j}$.

Therefore $थ$ is a minimal open cover of $F$ by Theorem 1.3. 
THEOREM 2.2. Let $\mathscr{D}=\cup_{i=1}^{\infty} \mathscr{D}_{i}$ be a sequence of discrete collections of nonempty closed subsets of a space $X$ with $\mathscr{D}_{i}=\{D(\alpha, i): \alpha \in A\}$. Let $D_{i}^{*}$ $=\cup_{\alpha \in A_{i}} D(\alpha, i)$ for each $i$ and $D^{*}=\cup_{i=1}^{\infty} D_{i}^{*}$.

If $\mathscr{Q}=\cup_{i=1}^{\infty} \mathscr{Q}_{i}=\cup_{i=1}^{\infty}\left\{U(\alpha, i): \alpha \in A_{i}\right\}$ is the union of a sequence of collections of open subsets of $X$ such that $D(\alpha, i) \subseteq U(\alpha, i)$ for each $\alpha \in A_{i}$ and each $i$, then $थ$ has an open refinement which covers $D^{*}$ minimally.

Proof. For each $i$ let $U_{i}^{*}=\cup_{\alpha \in A_{i}} U(\alpha, i)$. Now define $\mathscr{V}_{i}=\{V(\alpha, i): \alpha$ $\in A_{i}$ \} inductively by

$$
V(\alpha, i)=\left\{\begin{array}{l}
\varnothing \quad \text { if } D(\alpha, i)-\bigcup_{j<i} U_{j}^{*}=\varnothing, \\
U(\alpha, i)-\left[\left(\bigcup_{i<i} D_{j}^{*}\right) \cup\left(\underset{\beta \neq \alpha, \beta \in A_{i}}{\cup} D(\beta, i)\right)\right] \text { otherwise. }
\end{array}\right.
$$

It is easy to check that $\Upsilon_{i}$ is a minimal open cover of $D_{i}^{*}-\cup_{j<i} U_{j}^{*}$ for each $i$. Therefore, by Theorem 2.1, $\checkmark=\cup_{i=1}^{\infty} \mho_{i}$ has a refinement which covers $D^{*}$ minimally.

ConSTRUCTION THEOREM I. Let $\mathcal{G}=\cup_{i=1}^{\infty} \mathcal{S}_{i}$ be a weak $\bar{\theta}$-cover of a space $X$ with $\mathcal{S}_{i}=\left\{G(\alpha, i): \alpha \in A_{i}\right\}$. Now for each $i \geqslant 1$ and $j \geqslant 1$ define

$$
\begin{aligned}
P(i, j)=\left\{x \in X: \text { ord }\left(x, \mathcal{G}^{*}\right)<\right. & i \text { or ord }\left(x, \mathcal{G}^{*}\right)=i \\
& \text { and } \left.0<\operatorname{ord}\left(x, \mathcal{G}_{k}\right) \leqslant j \text { for some } k\right\} .
\end{aligned}
$$

If $\mathcal{G}$ has an open refinement $\mathfrak{}(i, j)$ which covers $P(i, j)$ minimally, then $\mathcal{G}$ has an open refinement $\mathfrak{V}(i, j+1)$ which covers $P(i, j+1)-V^{*}(i, j)$ minimally.

Proof. Suppose that $\mathcal{G}$ has an open refinement $\mathfrak{V}(i, j)$ which covers $P(i, j)$ minimally. If $P(i, j+1)-V^{*}(i, j)=\varnothing$ there is nothing to prove. Therefore suppose that $P(i, j+1)-V^{*}(i, j) \neq \varnothing$. Define

$$
\begin{aligned}
& H_{i}=\left\{x \in X: \text { ord }\left(x, \mathscr{Q}^{*}\right) \leqslant i\right\}, \\
& \mathscr{P}_{k}=\left\{B: B \subseteq A_{k} \text { and }|B|=j+1\right\}, \\
& S_{k}=\left\{x \in X: 0<\text { ord }\left(x, \mathscr{G}_{k}\right) \leqslant j+1\right\} .
\end{aligned}
$$

Now for each $k$ and $B \in \mathscr{B}_{k}$ let

$$
F(B, k)=\left[\cap_{\alpha \in B} G(\alpha, k)\right] \cap\left[G_{k}^{*} \cap H_{i} \cap S_{k}\right] \cap\left[X-V^{*}(i, j)\right] \text { and } \mathscr{F}_{k}
$$
$=\left\{F(B, k): B \in \mathscr{B}_{k}\right\}$.

We assert that $\mathscr{F}_{k}$ is a discrete collection of closed sets such that $\cup_{k=1}^{\infty} \mathscr{F}_{k}$ covers $P(i, j+1)-V^{*}(i, j)$.

Let $k$ be fixed and $x \in X$.

(1) If ord $\left(x, \mathcal{S}^{*}\right)<i$, then $x \in P(i, j)$. Thus $x \in V^{*}(i, j)$ which intersects no member of $\mathscr{F}_{k}$.

(2) If ord $\left(x, \mathcal{S}^{*}\right)>i$, then $X-H_{i}$ is a neighborhood of $x$ which intersects no member of $\mathscr{F}_{k}$.

(3) Suppose ord $\left(x, \mathcal{S}^{*}\right)=i$.

CASE I. If $x \notin G_{k}^{*}$, then $x$ belongs to exactly $i$ other members $\left\{G_{\alpha_{l}}^{*}: l\right.$ $=1,2, \ldots, i\}$ of $\mathcal{S}^{*}$. Hence $\bigcap_{l=1}^{i} G_{\alpha_{l}}^{*}$ is a neighborhood of $x$ which misses $G_{k}^{*} \cap H_{i}$ and thus misses each member of $\mathscr{F}_{k}$.

CASE II. Suppose $x \in G_{k}^{*}$.

(i) If ord $\left(x, \mathfrak{S}_{k}\right)<j+1$, then $x \in P(i, j)$ so that $V^{*}(i, j)$ is a neighborhood of $x$ which intersects no member of $\mathscr{F}_{k}$. 
(ii) If ord $\left(x, \mathscr{S}^{*}\right)>j+1$, then $x$ belongs to at least $j+2$ members of $\mathcal{G}_{k}$, say $G\left(\alpha_{l}, k\right)$ for $l=1,2, \ldots, j+2$. But $\cap j_{=1}^{+2} G\left(\alpha_{l}, k\right) \cap S_{k}=\varnothing$ so $x$ has a neighborhood which intersects no member of $\mathscr{F}_{k}$.

(iii) If ord $\left(x, \mathcal{G}_{k}\right)=j+1$, then $x$ belongs to exactly $j+1$ members of $\mathcal{S}_{k}, G\left(\alpha_{l}, k\right)$ for $l=1,2, \ldots, j+1$. However $\cap j_{=1}^{+1} G\left(\alpha_{l}, k\right)$ intersects only $F(B, k)$ where $B=\left\{\alpha_{1}, \alpha_{2}, \ldots, \alpha_{j+1}\right\}$.

Therefore, $\mathscr{F}_{k}$ is discrete. A similar argument shows that each member of $\mathscr{F}_{k}$ is closed.

Now let $V(B, k)$ be some member of $\mathfrak{S}_{k}$ which contains $F(B, k)$ if $F(B, k)$ $\neq \varnothing$ and let $\mathscr{V}_{k}=\left\{V(B, k): B \in \mathscr{B}_{k}\right\}$.

Thus $\mathfrak{V}=\cup_{i=1}^{\infty} \mathcal{V}_{i}$ and $\cup_{k=1}^{\infty} \mathscr{F}_{k}$ are sequences of collections satisfying the hypothesis of Theorem 2.2 above. Therefore $\mathcal{V}$ has an open refinement $\mathfrak{V}(i, j+1)$ which covers $F^{*}=\bigcup\left\{F: F \in \cup_{k=1}^{\infty} \mathscr{F}_{k}\right\}$ minimally. It is easy to see that $F^{*}=P(i, j+1)-V^{*}(i, j)$ so that the proof is complete.

THEOREM 2.4. Every weak $\bar{\theta}$-refinable space $X$ is irreducible.

Proof. Let $थ$ be an open cover of $X$. Since $X$ is weak $\bar{\theta}$-refinable, $\mathcal{Q}$ has a refinement $\mathcal{G}=\cup_{i=1}^{\infty} \mathcal{S}_{i}$ satisfying properties (i)-(iii) of Definition 1.1 above. Now define $P(1,0)=\varnothing, P(i, j)$ as in Construction Theorem I above for $i \geqslant 1, j \geqslant 1$ and $P(i, 0)=\cup_{l=1}^{i-1} \cup_{j=1}^{\infty} P(l, j)$ for $i \geqslant 2$. By induction and Construction Theorem $\mathrm{I}$, for each $i \geqslant 1$ and $j \geqslant 0$ there exists a refinement $\checkmark(i, j+1)$ of $\mathcal{G}$ which covers $P(i, j+1)-V^{*}(i, j)$ minimally. Let $F_{i}$ $=\cup_{j=1}^{\infty} P(i, j)$ for each $i$. Note that each $F_{i}$ is closed in $X$. By induction and repeated use of Theorem 2.1 above, there exists a sequence of open refinements $\left\{\mathscr{V}_{i}\right\}_{i=1}^{\infty}$ of $\mathcal{Q}$ such that $\Upsilon_{i}$ is a minimal cover of $F_{i}-\cup_{j<i} V_{j}^{*}$, where $V_{j}^{*}=\bigcup\left\{V: V \in \mathscr{V}_{j}\right\}$. Since $X=\cup_{i=1}^{\infty} F_{i}$, $Q$ has an open refinement which covers $X$ minimally by Theorem 2.1 again. Therefore, $X$ is irreducible.

3. Some applications. In [3] C. E. Aull introduced the notion of a distinguished point set and a $\delta \theta$-cover and thereby generalized a theorem of $\mathrm{G}$. Aquaro [1]. The following, through Theorem 3.4, are found in [3].

Let 2 be an open cover of a topological space $X$.

Definition 3.1. A set $M$ is distinguished with respect to $थ$ if for each pair $x, y \in M$ with $x \neq y$, then $x \in U \in \mathcal{Q} \Rightarrow y \notin U$.

LEMMA 3.2. For every subset $M$ of a space $X$ and every open (in $X$ ) cover $\mathcal{Q}$ of $M$, there exists a maximal distinguished set with respect to $थ$ which is discrete in थ*.

Definition 3.3. A space $X$ is called $\delta \theta$-refinable if every open cover $X$ has a refinement $\mathcal{G}=\cup_{i=1}^{\infty} \mathcal{S}_{i}$ satisfying:

(i) each $\mathcal{S}_{i}$ is an open cover of $X$;

(ii) for each $x \in X$ there exists an integer $n(x)$ such that ord $\left(x, \mathcal{S}_{n(x)}\right) \leqslant \aleph_{0}$.

THEOREM 3.4 (AULL). Every $\aleph_{1}$-compact, $\delta \theta$-refinable space is Lindelöf.

We now consider the following modifications of Definition 3.3 above.

Definition 3.5. (1) A space $X$ is called weak $\overline{\delta \theta}$-refinable if every open cover of $X$ has a refinement $\mathcal{G}=\cup_{i=1}^{\infty} \varrho_{i}$ satisfying:

(i) each $\mathcal{G}_{i}$ is a collection of open subsets of $X$; 
(ii) for each $x \in X$ there exists an integer $n(x)$ such that $0<$ ord $\left(x, \mathcal{G}_{n(x)}\right)$ $\leqslant \aleph_{0}$

(iii) $\left\{G_{i}^{*}=\cup\left\{G: G \in \mathcal{G}_{i}\right\}\right\}_{i=1}^{\infty}$ is point finite.

(2) A space $X$ is called weak $\delta \theta$-refinable if every open cover of $X$ has a refinement $\mathcal{G}=\cup_{i=1}^{\infty} \mathcal{S}_{i}$ satisfying (i) and (ii) above. We will naturally call such covers described above as weak $\overline{\delta \theta}$-covers and weak $\delta \theta$-covers respectively.

REMARK. In (1) above the condition that " $\mathcal{G}_{i}$ covers $X$ " is relaxed but the fact that the "levels" $\left\{G_{i}^{*}\right\}_{i=1}^{\infty}$ be point finite is added. For (2) this last condition is dropped.

Construction Theorem II. Let $\mathcal{G}=\cup_{i=1}^{\infty} \mathcal{G}_{i}$ be a weak $\overline{\delta \theta}$-cover of an $\boldsymbol{\aleph}_{1}$ compact space $X$. Define for each $i$,

$$
P_{i}=\left\{x \in X: \text { ord }\left(x, \mathcal{G}^{*}\right) \leqslant i\right\} .
$$

If $P_{i}$ is covered by a countable subfamily of $\mathcal{G}$, then so is $P_{i+1}$. G.

Proof. Suppose that $P_{i}$ is covered by a countable subfamily $\checkmark=\left\{V_{i}\right\}_{i=1}^{\infty}$ of

Define $\mathscr{B}_{i+1}=\{B: B$ is a subset of the positive integers with $|B|=i+1\}$ and let $F(B)=\left(P_{i+1}-V^{*}\right) \cap\left(\cap_{k \in B} G_{k}^{*}\right)$ for $B \in \mathscr{B}_{i+1}$.

By an argument similar to that used in Construction Theorem I, $\mathscr{F}$ $=\left\{F(B): B \in \mathscr{B}_{i+1}\right\}$ is a discrete collection of closed subsets of $X$. By Lemma 3.2 , for each $k \in B, F(B)$ contains a maximal distinguished subset $M(B, k)$ with respect to $\mathcal{G}_{k}$, where $M(B, k)$ consists of points of countable order with respect to $\mathcal{G}_{k}$. Since $X$ is $\boldsymbol{\kappa}_{1}$-compact, $M(B, k)$ is countable for each $k \in B$. Therefore, $F(B)$ is covered by a countable subfamily of $\mathcal{G}$ and hence so is $F^{*}$. Therefore, $P_{i+1}$ is covered by a countable subfamily of $\mathcal{G}$.

THEOREM 3.6. Let $X$ be an $\aleph_{1}$-compact space. If $X$ is weak $\overline{\delta \theta}$-refinable then $X$ is Lindelöf.

Proof. The proof follows immediately from induction and Construction Theorem II above.

CoRollaRY 3.7. Let $X$ be an $\aleph_{1}$-compact and countably metacompact space. If $X$ is weak $\overline{\delta \theta}$-refinable, then $X$ is metacompact and hence irreducible.

It is now natrual to ask whether Theorem 3.6, and hence Corollary 3.7, is true for the class of weak $\delta \theta$-refinable spaces. If so, this would provide a result analogous to that of Worrell and Wicke [12] which states that countably compact, weak $\delta \theta$-refinable spaces are compact.

This problem seems to be rather difficult; however, the techniques used above will apply if the countably metacompactness condition is strengthened to perfect.

Definition 3.8. A space $X$ is perfect if every closed subset of $X$ is a $G_{\delta}$-set.

REMARK. Bennett and Lutzer [4] have shown that perfect weak $\theta$-refinable spaces are subparacompact and hence irruducible. It is not known whether a perfect, weak $\delta \theta$-refinable space is subparacompact or even $\theta$-refinable. Thus it is an open question whether or not perfect, weak $\delta \theta$-refinable spaces are irreducible. In the presence of $\aleph_{1}$-compactness however, the situation is much nicer. 
THEOREM 3.9. Let $X$ be a perfect, $\aleph_{1}$-compact space. Then every weak $\delta \theta$-cover of $X$ has a countable subcover. ${ }^{1}$

Proof. Let $\mathcal{G}=\cup_{i=1}^{\infty} \mathcal{S}_{i}$ be a weak $\delta \theta$-cover of $X$. Since $X$ is perfect, for each $G_{i}^{*}=\cup\left\{G: G \in \mathcal{G}_{i}\right\}$ there exists a sequence of closed sets $\{F(i, j)\}_{j=1}^{\infty}$ such that $\Theta_{i}^{*}=\cup_{j=1}^{\infty} F(i, j)$.

If $\mathcal{G}$ does not have a countable subcover, then there exists some $i_{0}$ and $j_{0}$ such that no countable subfamily of $\mathcal{S}_{i_{0}}$ covers $F\left(i_{0}, j_{0}\right)$. This is a contradiction, however, since $\mathcal{S}_{i_{0}}$ is a point countable open cover of the $\boldsymbol{\kappa}_{1}$-compact space $F\left(i_{0}, j_{0}\right)$. Therefore $\mathcal{G}$ must have a countable subcover.

THEOREM 3.10. Every perfect, $\boldsymbol{\aleph}_{1}$-compact weak $\delta \theta$-refinable space is hereditarily Lindelöf.

Proof. It is easy to show that for perfect spaces, the properties of $\boldsymbol{\aleph}_{1}$ compactness and weak $\delta \theta$-refinability are hereditary. The result now follows by Theorem 3.9 above.

COROLlaRY 3.11. Every perfect, $\boldsymbol{\aleph}_{1}$-compact weak $\delta \theta$-refinable space is irreducible.

4. Some problems. A number of new problems have arisen from the previous investigations. These are listed here for the benefit of the reader.

Problem 1. Is every $\delta \theta$-refinable space, weak $\overline{\delta \theta}$-refinable? The author [11] has shown that $\theta$-refinable spaces are weak $\bar{\theta}$-refinable. An affirmative answer here would generalize Aull's result.

Problem 2. What properties do perfect, weak $\delta \theta$-refinable spaces possess? In particular, is every perfect, weak $\delta \theta$-refinable space weak $\overline{\delta \theta}$-refinable?

Problem 3. Can the condition of being perfect in Theorem 3.9 above be weakened to countably metacompactness?

Problem 4. What conditions in the above results can be dropped if $X$ is normal? ble?

Problem 5. Are countably metacompact, weak $\theta$-refinable spaces irreduci-

\section{REFERENCES}

1. G. Aquaro, Point countable open coverings in countably compact spaces, General Topology and its Relations to Modern Analysis and Algebra. II (Proc. Second Prague Topological Sympos. 1966), Academia, Prague, 1967, pp. 39-41.

2. R. Arens and J. Dugundji, Remark on the concept of compactness, Portugal. Math. 9 (1950), 141-143. MR 12, 434.

3. C. Aull, A generalization of a theorem of Aquaro, Bull. Austral. Math. Soc. 9 (1973), 105-108.

4. H. R. Bennett and D. J. Lutzer, A note on weak $\theta$-refinability, General Topology and Appl. 2 (1972), 49-54. MR 46 \#853. 264.

5. R. H. Bing, Metrization of topological spaces, Canad. J. Math. 3 (1951), 175-186. MR 13,

6. J. Boone, On irreducible spaces, Bull. Austral. Math. Soc. 12 (1975), 143-148.

7. — On irreducible spaces. II, Pacific J. Math. (to appear).

8. D. K. Burke, A note on R. H. Bing's example G, (Proc. Virginia Polytechnic Inst. and State

\footnotetext{
${ }^{1}$ The author would like to thank the referee for his suggestions in simplifying the proof of this theorem.
} 
Univ. Topology Conf.), Lecture Notes in Math, vol. 375, Springer-Verlag, New York and Berlin, 1973, pp. 47-52.

9. U. Christian, $A$ note on the relation between Lindelöf and $\aleph_{1}$-compact space, Comment. Math. Proc. Math. 16 (1972), 215-217.

10. Concerning certain minimal cover refinable spaces, Fund. Math. 76 (1972), 213-222.

11. J. C. Smith, Properties of weak $\bar{\theta}$-refinable spaces, Proc. Amer. Math. Soc. 53 (1975), 511-517.

12. J. M. Worrell, Jr. and H. H. Wicke, Characterizations of developable topological spaces, Canad. J. Math. 17 (1965), 820-830. MR 32 \#427.

Department of Mathematics, Virginia Polytechnic Institute and State University, BLACKSBURG, VIRGINIA 24061 